\title{
Canonical Maps Between Semidirect Products with Applications to Elasticity and Superfluids
}

\author{
Boris A. Kupershmidt ${ }^{1}$ and Tudor Ratiu ${ }^{2}$ \\ Department of Mathematics, University of Michigan, Ann Arbor, MI 48109, USA
}

\begin{abstract}
It is shown that two canonical maps arising in the Poisson bracket formulations of elasticity and superfluids are particular instances of general canonical maps between duals of semidirect product Lie algebras.
\end{abstract}

\section{Introduction}

In the last years many models in classical physics have been shown to possess Poisson structures. In many examples this Poisson structure is the canonical one on the dual of a Lie algebra, sometimes supplemented by a two-cocycle. It turns out that in almost all such cases, the Lie algebra in question is a semidirect product.

Quite often the same physical system allows descriptions in different sets of variables, thus obtaining two Poisson structures for the same model. These structures are not equivalent but connected. Such double descriptions commonly occur, e.g., in systems coupled to the magnetic field by introducing magnetic potentials; magnetohydrodynamics is such a system. The relation between the two descriptions, when linear, is produced by a Lie algebra homomorphism (like in the above-mentioned magnetic case of magnetohydrodynamics, see $[5,8]$ ). In this case the dual map of the Lie algebra homomorphism is naturally canonical between the two Poisson structures. However, it was recently $[5,6]$ observed experimentally that in two physical models, elastodynamics and superhydrodynamics, the transformation between the two Poisson structures, even though non-linear, is still canonical. We hasten to emphasize that this is not the standard case in the theory of finite dimensional Lie algebras: given two general Lie algebras $\mathfrak{5}$ and $\mathfrak{6}$, there are no natural non-linear canonical maps from $\mathfrak{G}^{*}$ to $\mathfrak{H}^{*}$. The problem of interpretation of the above-mentioned non-linear canonical maps is the topic of this paper. Our explanation turns out to be very natural and simple (see, e.g., Theorem 3.5 below): canonical maps with range the dual of a Lie algebra are realized as momentum maps. This underlying philosophy is closely related to [8].

1 Partially supported by NSF Grant MCS-8003104

2 Partially supported by NSF Grant MCS-8106142 
Let us describe briefly our set-up. In the final analysis, a simplified version of the problem boils down to the following question. Let $\mathfrak{5}, \mathfrak{H}_{1}, \mathfrak{S}_{2}$ be Lie algebras and $\phi:\left(\tilde{\mathfrak{b}} \rightarrow \operatorname{Der}\left(\mathfrak{S}_{1}\right) \quad \psi:\left(\mathfrak{5} \rightarrow \operatorname{Der}\left(\mathfrak{H}_{2}\right)\right.\right.$ be Lie algebra homomorphisms, where Der denotes the Lie algebra of derivations. Let $f: \mathfrak{H}_{1}^{*} \rightarrow \mathfrak{H}_{2}^{*}$ be a map. Under what conditions is the induced map id $\times f:\left(\mathfrak{6} \times \mathfrak{S}_{1}\right)^{*} \rightarrow\left(\mathfrak{G} \times \mathfrak{H}_{2}\right)^{*}$ between the duals of semidirect products canonical? Our answer, given in different versions needed for the treatment of the examples, is that $f: \mathfrak{S}_{1}^{*} \rightarrow \mathfrak{S}_{2}^{*}$ must be a canonical map of Poisson manifolds compatible with the actions of (5 (see Theorem 3.3).

The plan of the paper is the following. Section 2 recalls the definitions and formulas relevant for semidirect products of Lie groups and algebras. Section 3 describes the general set-up for getting non-linear canonical maps between semidirect products and gives the general theorems to be used in the next section. Section 4 starts with four theoretical examples which together with the main theorems of the previous section enable us to show that the non-linear canonical maps coming up in elastodynamics and superhydrodynamics are particular instances of our general theorems.

Throughout the paper we employ the following conventions and notations. For a manifold $P, \mathscr{F}(P), \mathscr{X}(P)$ denote the ring of functions and the Lie algebra of vector fields respectively. A Poisson bracket on $P$ is a multiplication $\{$,$\} on$ $\mathscr{F}(P)$ making $(\mathscr{F}(P),\{\}$,$) into a Lie algebra and such that the map f \mapsto X_{f} \in \mathscr{X}(P)$, $X_{f}(g):=\{f, g\}$, is a Lie algebra homomorphism of $\mathscr{F}(P)$ into $\mathscr{X}(P)$, i.e. $X_{\{f, g\}}=$ $\left[X_{f}, X_{q}\right]$. A manifold $P$ endowed with a Poisson bracket is called a Poisson manifold. A map $\alpha:\left(P_{1},\{,\}_{1}\right) \rightarrow\left(P_{2},\{,\}_{2}\right)$ between Poisson manifolds is called canonical, if

$$
\alpha^{*}\{f, g\}_{2}=\left\{\alpha^{*} f, \alpha^{*} g\right\}_{1}
$$

for any $f, g \in \mathscr{F}\left(P_{2}\right)$, where the upper star denotes the pull-back operation.

A Lie group action on a manifold $P$ is a group homomorphism $\Phi: G \rightarrow \operatorname{Diff}(P)$, where Diff $(P)$ denotes the group of diffeomorphisms of $P$, such that the map $(g, p) \mapsto \Phi_{g}(p)$ is smooth. If $P$ is a Poisson manifold, $\Phi$ is called canonical if all the diffeomorphisms $\Phi_{g}, g \in G$, are canonical maps of $P$. A Lie algebra action on a manifold $P$ is a Lie algebra homomorphism $\phi:(5 \rightarrow \mathscr{X}(P)$ such that the map $(\xi, p) \mapsto \phi(\xi)(p)$ is smooth. If ( 5 happens to be the (left) Lie algebra of a Lie group $G$ acting on $P$, then $\phi=-\Phi^{\prime}$, where the upper prime denotes the Lie algebra homomorphism induced by $\Phi .{ }^{1}$ If $P$ is a Poisson manifold, the Lie algebra action $\phi$ is said to be canonical if for any $\xi \in \mathfrak{G}$ and $f_{1}, f_{2} \in \mathscr{F}(P)$,

$$
\phi(\xi)\left\{f_{1}, f_{2}\right\}=\left\{\phi(\xi) f_{1}, f_{2}\right\}+\left\{f_{1}, \phi(\xi) f_{2}\right\} .
$$

If the Lie group $G$ with Lie algebra $(5$ acts canonically on the Poisson manifold $P$, a momentum mapping $J: P \rightarrow \mathfrak{G}^{*}$ is a map satisfying

$$
\phi(\xi)=X_{\hat{J}(\xi)}
$$

for all $\xi \in \mathfrak{F}$, where $\hat{J}(\xi) \in \mathscr{F}(P)$ is defined by $\hat{J}(\xi)(p)=\langle J(p), \xi\rangle$, where $\langle$, $\rangle$ denotes the pairing between $\mathfrak{G}^{*}$ and $\mathfrak{6} . J$ is said to be equivariant, if

1 The reason for the minus in front of $\Phi^{\prime}$ is due to the fact that $\mathscr{X}(P)$ is the right Lie algebra of Diff $(P)$. (See [1], ex. 4.1G, page 274.) 


$$
J \circ \Phi_{g}=\operatorname{Ad}_{g^{-1}}^{*} \circ J
$$

for all $g \in G$; here $\mathrm{Ad}_{g}:\left(\mathfrak{b} \rightarrow \mathfrak{b}\right.$ denotes the adjoint action of $G$ on $\left(\mathfrak{5}\right.$ and $\mathrm{Ad}_{g}^{*}:\left(\mathfrak{5}^{*} \rightarrow \mathfrak{F}^{*}\right.$ is its dual map. If we deal with a canonical Lie algebra action $\phi$ of $\mathfrak{G}$ on $P$, the definition of the momentum mapping is unchanged, but equivariance is replaced by

$$
T_{p} J(\phi(\xi)(p))=(\operatorname{ad} \xi)^{*}(J(p))
$$

for all $\xi \in \mathfrak{G}, p \in P$; here $T_{p} J: T_{p} P \rightarrow \mathfrak{G}^{*}$ denotes the tangent map (differential) of $J$ at $p \in P$. (The formula has no minus signs since $\phi=-\Phi^{\prime}$.) Lie group (algebra) actions on a Poisson manifold admitting equivariant momentum maps are called Hamiltonian actions.

The dual $\mathfrak{5}^{*}$ of a Lie algebra $(5$ is a Poisson manifold with respect to the LiePoisson bracket given by

$$
\{f, g\}(\mu)=\left\langle\mu,\left[\frac{\delta f}{\delta \mu}, \frac{\delta g}{\delta \mu}\right]\right\rangle,
$$

for $\mu \in \mathfrak{G}^{*}$ and $f, g$ functions on $\mathfrak{G}^{*}$; here $\langle$,$\rangle denotes the pairing between \mathfrak{G}$ and $\mathfrak{G}^{*}$. The "functional derivative" $\delta f / \delta \mu \in \mathfrak{G}$ is the derivative $D f(\mu)$ regarded as an element of 6 rather than $5^{* *}$, i.e.

$$
D f(\mu) \cdot v=\left\langle v, \frac{\delta f}{\delta \mu}\right\rangle
$$

for $\mu, v \in \mathfrak{G}^{*}$. For infinite dimensional $\mathfrak{5}$, the pairing is with respect to a weakly nondegenerate form and the existence of $\delta f / \delta \mu$ is a bona fide hypothesis on $f$. The same formula defines the Lie-Poisson bracket on polynomial functions on the dual of a Lie algebra over any ring.

The Hamiltonian vector field defined by the function $h$ on $\mathfrak{b}^{*}$ is given by

$$
X_{h}(\mu)=\operatorname{ad}\left(\frac{\delta h}{\delta \mu}\right)^{*}(\mu)
$$

where $\operatorname{ad}(\xi) \cdot \eta=[\xi, \eta]$ is the adjoint action of $\left(\mathfrak{5}\right.$ on $\left(\mathfrak{5}\right.$ and $(\operatorname{ad}(\xi))^{*}\left(\mathfrak{5}^{*} \rightarrow \mathfrak{6}^{*}\right.$ its dual map.

The following standard fact of great use in the paper can be found in the symplectic context in e.g. $[1,3,9]$.

Proposition 1.1. Let $P$ be a Poisson manifold and $\varphi:(\tilde{\mathfrak{G}} \rightarrow \mathscr{X}(P)$ a canonical Lie algebra action. The following are equivalent:

(i) the action is Hamiltonian;

(ii) there exists a Lie algebra homomorphism $\psi:\left(\mathfrak{G} \rightarrow \mathscr{F}(P)\right.$ such that $\varphi(\xi)=X_{\psi(\xi)}$ for all $\xi \in(\mathfrak{5}$;

(iii) there exists a ring and Lie algebra homomorphism $\chi: \mathscr{F}\left(\mathfrak{G}^{*}\right) \rightarrow \mathscr{F}(P)$ such that $X_{(\alpha \cdot j)(\xi)}=\varphi(\xi)$ for all $\xi \in\left(\mathfrak{G}\right.$, where $j:\left(\mathfrak{H} \rightarrow \mathscr{F}\left(\mathfrak{G}^{*}\right)\right.$ is the Lie algebra homomorphism given by $j(\xi)(\mu)=\langle\mu, \xi\rangle$.

In fact, if $J$ is the momentum map of the action $\varphi$, then $\psi=\hat{J}$ and $\chi=J^{*}$.

A second standard fact to be used later on is the following. 
Proposition 1.2. Let $\mathbf{6}, \mathfrak{S}$ be Lie algebras and $\alpha:(\mathfrak{5} \rightarrow \mathfrak{S}$ a linear map. The dual map $\alpha^{*}: \mathfrak{S}^{*} \rightarrow \mathfrak{5}^{*}$ is canonical if and only if $\alpha$ is a Lie algebra homomorphism.

For a study of the local structure of Poisson manifolds the reader is referred to [10].

\section{Semidirect Products of Lie Groups and Lie Algebras}

Let $G, H$ be Lie groups with Lie algebras $(\mathfrak{G}$ and $\mathfrak{S}$ respectively. Denote by $\operatorname{Aut}(H)$ the Lie group of automorphisms of $H$ and by $\operatorname{Der}(\mathfrak{S})$ the Lie algebra of derivations of $\mathfrak{S}$.

Let $\Phi: G \rightarrow \operatorname{Aut}(H)$ be a Lie group homomorphism. The semidirect product $G \underset{\Phi}{\times} H$ of $G$ with $H$ by $\Phi$ is a Lie group with underlying manifold $G \times H$, composition law

$$
\left(g_{1}, h_{1}\right)\left(g_{2}, h_{2}\right)=\left(g_{1} g_{2}, h_{1} \Phi\left(g_{1}\right)\left(h_{2}\right)\right)
$$

identity element $(e, e)$, and inverse $(g, h)^{-1}=\left(g^{-1}, \Phi\left(g^{-1}\right)\left(h^{-1}\right)\right)$.

The homomorphism $\Phi$ induces a Lie algebra homomorphism $\phi:(\mathfrak{b} \rightarrow \operatorname{Der}(\mathfrak{S})$ in the following way. For every $g \in G, \Phi(g): H \rightarrow H$ is an automorphism which thus induces a Lie algebra automorphism $\tilde{\Phi}(g):=\Phi(g)^{\prime}: \mathfrak{j} \rightarrow \mathfrak{H}$. In this way $\tilde{\Phi}: G \rightarrow$ Aut $(\mathfrak{S})$ becomes a Lie group homomorphism whose induced Lie algebra homomorphism $\phi:=\widetilde{\Phi}^{\prime}:(\mathfrak{5} \rightarrow \operatorname{Der}(\mathfrak{G})$ allows one to define the semidirect product $\mathfrak{6} \times \mathfrak{S}$ as the Lie algebra with underlying vector space $65 \mathfrak{5}$ and bracket

$$
\left[\left(\xi_{1}, \eta_{1}\right),\left(\xi_{2}, \eta_{2}\right)\right]=\left(\left[\xi_{1}, \xi_{2}\right], \phi\left(\xi_{1}\right) \eta_{2}-\phi\left(\xi_{2}\right) \eta_{1}+\left[\eta_{1}, \eta_{2}\right]\right) \text {. }
$$

where $\xi_{1}, \xi_{2} \in \mathfrak{G}, \eta_{1}, \eta_{2} \in \mathfrak{H}$. It is well known that the Lie algebra of $\underset{\Phi}{\times} H$ is $\underset{\Phi}{ } \times \mathfrak{S}$ (see [14]).

Let $\mathscr{L}($ Aut $(H))$ denote the Lie algebra of Aut $(H)$. To identify elements of $\mathscr{L}($ Aut $(H))$, let $c:(-\varepsilon, \varepsilon) \rightarrow \operatorname{Aut}(H)$ be a smooth curve with $c(0)$ the identity map of $H$. Then for any $h \in H, d /\left.d t\right|_{t=0} c(t)(h) \in T_{h} H$, i.e. $c^{\prime}(0)$ defines a vector field on $H$ by $h \mapsto c^{\prime}(0)(h)$. Thus, if $\mathscr{X}(H)$ denotes the vector space of all vector fields on $H, \mathscr{L}(\operatorname{Aut}(H)) \subset \mathscr{X}(H)$. It can be shown from the fact that $c(t) \in \operatorname{Aut}(H)$, that $c^{\prime}(0)(e)=0$, but this fact will not be used in the sequel. If $\Phi^{\prime}:(5 \rightarrow \mathscr{L}$ (Aut $(H))$ is the Lie algebra homomorphism induced by $\Phi$, we have $\Phi^{\prime}(\xi) \in \mathscr{X}(H)$ for all $\xi \in(5$. With these notations, the adjoint action of $G \underset{\Phi}{\times} H$ on $\mathfrak{G} \underset{\phi}{\times \mathfrak{H}}$ is given by

$$
\operatorname{Ad}_{(g, h)}(\xi, \eta)=\left(\operatorname{Ad}_{g} \xi,\left(\operatorname{Ad}_{h} \circ \tilde{\Phi}(g)\right)(\eta)+T_{h^{-1}} L_{h}\left(\left[\Phi^{\prime}\left(\operatorname{Ad}_{g} \xi\right)\right]\left(h^{-1}\right)\right)\right),
$$

where $\xi \in \mathfrak{G}, \eta \in \mathfrak{Y}, g \in G, h \in H$ and $T_{h^{-1}} L_{h}: T_{h^{-1}} H \rightarrow \mathfrak{G}$ is the derivative of the left translation $L_{h}$ on $H$ at $h^{-1} \in H$. To compute the coadjoint action, more notation is needed. For any Lie algebra homomorphism $F:(5 \rightarrow \mathscr{L}($ Aut $(H))$ and any $h \in H$, denote $F^{\vee}(h):\left(\mathfrak{G} \rightarrow T_{h} H\right.$ the linear map given by $F^{\vee}(h)(\xi)=F(\xi)(h)$. Thus the dual map $F^{\vee}(h)^{*}: T_{h}^{*} H \rightarrow\left(\mathfrak{b}^{*}\right.$. With this notation the coadjoint action is given by

$$
\begin{gathered}
\operatorname{Ad}_{(g, h)^{-1}}^{*}(\mu, v)=\left(\operatorname{Ad}_{g^{-1}}^{*} \mu+\left(\Phi^{\prime} \circ \operatorname{Ad}_{g^{-1}}\right)^{\vee}\left(\Phi\left(g^{-1}\right)\left(h^{-1}\right)\right)^{*}\left(T_{\Phi\left(g^{-1}\right)(h)} L_{\Phi\left(g^{-1}\right)\left(h^{-1}\right)}\right)^{*} v,\right. \\
\left.\tilde{\Phi}\left(g^{-1}\right)^{*} \operatorname{Ad}_{\Phi\left(g^{-1}\right)\left(h^{-1}\right)}^{*} v\right) .
\end{gathered}
$$


The Lie-Poisson bracket of $F_{1}, F_{2}:(\mathfrak{G} \times \mathfrak{S})^{*} \rightarrow \mathbb{R}$, becomes with the use of $(2.2)$

$$
\begin{aligned}
\left\{F_{1}, F_{2}\right\}(\mu, v)= & \left\langle\mu,\left[\frac{\delta F_{1}}{\delta \mu}, \frac{\delta F_{2}}{\delta \mu}\right]\right\rangle+\left\langle v, \phi\left(\frac{\delta F_{1}}{\delta \mu}\right) \frac{\delta F_{2}}{\delta v}\right\rangle \\
& -\left\langle v, \phi\left(\frac{\delta F_{2}}{\delta \mu}\right) \frac{\delta F_{1}}{\delta v}\right\rangle+\left\langle v,\left[\frac{\delta F_{1}}{\delta v}, \frac{\delta F_{2}}{\delta v}\right]\right\rangle,
\end{aligned}
$$

where the functional derivatives $\delta F_{1} / \delta \mu \in \mathfrak{G}, \delta F_{1} / \delta \nu \in \mathfrak{H}$ for $\mu \in \mathfrak{G}^{*}, v \in \mathfrak{S}^{*}$. The

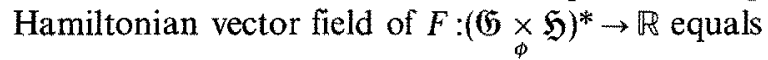

$$
X_{F}(\mu, v)=\left(\operatorname{ad}\left(\frac{\delta F}{\delta \mu}\right)^{*} \mu-\phi_{\delta F / \delta v}^{*} v, \phi\left(\frac{\delta F}{\delta \mu}\right)^{*} v+\operatorname{ad}\left(\frac{\delta F}{\delta v}\right)^{*} v\right),
$$

where $\mu \in \mathfrak{G}^{*}, v \in \mathfrak{I}^{*}$, and for $\eta \in \mathfrak{S}, \phi_{\eta}:\left(\mathfrak{5} \rightarrow \mathfrak{H}\right.$ is given by $\phi_{\eta}(\xi)=\phi(\xi) \cdot \eta$.

Let us specialize the foregoing definitions and formulas to the case $H=V$, a vector space, regarded as an abelian Lie group under addition, and $\Phi: G \rightarrow \operatorname{Aut}(V)$ a linear representation. Since the Lie algebra of $V$ is $V$ itself, $\widetilde{\Phi}=\Phi$, and $\phi=\Phi^{\prime}$ : $G \rightarrow \operatorname{End}(V)$, where End $(V)$ denotes the algebra of endomorphisms of $V$. The composition law in $G \underset{\Phi}{\times} V$ is hence

$$
\left(g_{1}, v_{1}\right)\left(g_{2}, v_{2}\right)=\left(g_{1} g_{2}, v_{1}+\Phi\left(g_{1}\right) v_{2}\right)
$$

the bracket in $\mathfrak{6} \underset{\phi}{\times} V$ is

$$
\left[\left(\xi_{1}, v_{1}\right),\left(\xi_{2}, v_{2}\right)\right]=\left(\left[\xi_{1}, \xi_{2}\right], \phi\left(\xi_{1}\right) v_{2}-\phi\left(\xi_{2}\right) v_{1}\right),
$$

and the adjoint and coadjoint actions are given by

$$
\begin{aligned}
\operatorname{Ad}_{(g, u)}(\xi, v) & =\left(\operatorname{Ad}_{g} \xi, \Phi(g) v-\phi\left(\operatorname{Ad}_{g} \xi\right) u\right), \\
\operatorname{Ad}_{(g, u)^{-1}}^{*}(\mu, a) & =\left(\operatorname{Ad}_{g^{-1}}^{*} \mu+\phi_{u}^{*} \Phi\left(g^{-1}\right)^{*} a, \Phi\left(g^{-1}\right)^{*} a\right),
\end{aligned}
$$

where $g \in G, u, v \in V, \xi \in \mathfrak{G}, \mu \in \mathfrak{G}^{*}, a \in V^{*}$. Finally, the Lie-Poisson bracket and Hamiltonian vector field take the form

$$
\begin{aligned}
\left\{F_{1}, F_{2}\right\}(\mu, a)=\left\langle\mu_{y}\left[\frac{\delta F_{1}}{\delta \mu}, \frac{\delta F_{2}}{\delta \mu}\right]\right\rangle+\left\langle a, \phi\left(\frac{\delta F_{1}}{\delta \mu}\right) \frac{\delta F_{2}}{\delta a}\right\rangle-\left\langle a, \phi\left(\frac{\delta F_{2}}{\delta \mu}\right) \frac{\delta F_{1}}{\delta a}\right\rangle \\
X_{F}(\mu, a)=\left(\operatorname{ad}\left(\frac{\delta F}{\delta \mu}\right)^{*} \mu-\phi_{\delta F / \delta a}^{*} a, \phi\left(\frac{\delta F}{\delta \mu}\right)^{*} a\right)
\end{aligned}
$$

where $\mu \in \mathfrak{G}^{*}, a \in V^{*}, F_{1}, F_{2}, F \in \mathscr{F}\left((\underset{\phi}{\times} V)^{*}\right)$.

\section{Canonical Maps Associated to Semidirect Products}

Let $G$ be a Lie group with Lie algebra $(\mathfrak{6}, V, W$ vector spaces and $\Phi: G \rightarrow \operatorname{Aut}(V)$, $\Psi: G \rightarrow \operatorname{Aut}(W)$ two linear representations. Let $f: V^{*} \rightarrow W^{*}$ be any map compatible with the representations, i.e.

$$
f \circ \Phi(g)^{*}=\Psi(g)^{*} \circ f
$$


for all $g \in G$. If instead of the group representations one works with Lie algebra representations, this relation is replaced by

$$
D f(a)\left(\phi(\xi)^{*} a\right)=\psi(\xi)^{*}(f(a))
$$

for all $\xi \in\left(\mathfrak{G}, a \in V^{*}\right.$, where $\phi:(\mathfrak{G} \rightarrow \operatorname{End}(V), \psi:(\mathfrak{G} \rightarrow \operatorname{End}(W)$ are Lie algebra representations. If $G$ is the Lie algebra of $G$, then $\phi=\Phi^{\prime}, \psi=\Psi^{\prime}$. Guided by the formula (2.8) of the coadjoint representation, define an action of $G \underset{\psi}{\times} W$ on $((6) \underset{\phi}{\times} V)^{*}$ by

$$
(g, w) \cdot(\mu, a)=\left(\operatorname{Ad}_{g-1}^{*} \mu+\psi_{w}^{*} \Psi\left(g^{-1}\right)^{*} f(a), \Phi\left(g^{-1}\right)^{*} a\right)
$$

for $g \in G, w \in W, \mu \in \mathfrak{G}^{*}, a \in V^{*}$; recall that $\psi_{w}:\left(\mathfrak{G} \rightarrow W\right.$ is given by $\psi_{w}(\xi)=\psi(\xi) \cdot w$. It can be shown quite laboriously that this action is canonical. We shall not do this here since it will follow trivially later on. It is convenient to give a different formula for this action.

Lemma. For $w \in W, a \in V^{*}$ we have

$$
\psi_{w}^{*} f(a)=\phi_{w \circ D f(a)}^{*} a
$$

where on the right hand side $w$ is thought of as an element of $W^{* *}$.

Proof. For $\xi \in \mathbb{6}$ we have

$$
\left\langle\psi_{w}^{*} f(a) ; \zeta\right\rangle=f(a) \cdot \psi(\xi) w=\psi(\xi)^{*} f(a) \cdot w^{(3.2)}=D f(a)\left(\phi(\xi)^{*} f(a)\right) \cdot w
$$

Thinking now of $w$ as a linear functional on $W^{*}$ this equals $(w \circ D f(a)) \cdot \phi(\xi)^{*} f(a)$. Thus $w \circ D f(a) \in W^{* *}$; denoting by the same symbol the corresponding element of $V$, the above equals

$$
f(a) \cdot \phi(\xi)(w \circ D f(a))=f(a) \cdot \phi_{w \circ D f(a)}(\xi)=\left\langle\phi_{w \circ D f(a)}^{*} f(a), \xi\right\rangle .
$$

Replacing $a$ by $\Phi\left(g^{-1}\right)^{*} a$ in (3.4) and taking into account (3.1), we get $\psi_{w}^{*} \Psi\left(g^{-1}\right)^{*} f(a)=\phi_{\left.w \circ D f\left(\Phi^{-1}\right)^{*} a\right)}^{*} \Phi\left(g^{-1}\right)^{*} a$. Thus an alternate expression of (3.3) is

$$
(g, w) \cdot(\mu, a)=\left(\mathrm{Ad}_{g-1}^{*} \mu+\phi_{w \circ D f\left(\phi g^{-1}\right)^{*} a \mid}^{*} \Phi\left(g^{-1}\right)^{*} a, \Phi\left(g^{-1}\right)^{*} a\right) .
$$

The corresponding Lie algebra action of $\left(5 \times W\right.$ on $\left((5 \underset{\phi}{\times} V)^{*}\right.$ thus has the expression

$$
\begin{aligned}
(\xi, w) \cdot(\mu, a) & =\left((\operatorname{ad} \xi)^{*} \mu-\psi_{w}^{*} f(a), \phi(\xi)^{*} a\right) \\
& =\left((\operatorname{ad} \xi)^{*} \mu-\phi_{w \circ D f(a)}^{*} a, \phi(\xi)^{*} a\right)
\end{aligned}
$$

for $\xi \in\left(\mathfrak{G}, w \in W, \mu \in \mathfrak{G}^{*}, a \in V^{*}\right.$; recall that the Lie algebra action is minus the derivative of the Lie group action.

Let us compute the momentum map $J:(\mathfrak{G} \underset{\phi}{\times} V)^{*} \rightarrow(\mathfrak{G} \underset{\psi}{\times} W)^{*}$ of this action. By definition we must have

$$
X_{\hat{J}(\xi, w)}(\mu, a)=(\xi, w) \cdot(\mu, a)
$$


for all $\xi \in \mathfrak{G}, w \in W, \mu \in \mathfrak{G}^{*}, a \in V^{*}$. A comparison between (3.6) and (2.10) shows that

$$
\frac{\delta \widehat{J}(\xi, w)}{\delta \mu}=\xi, \frac{\delta \hat{J}(\xi, w)}{\delta a}=w \circ D f(a)=D(w \circ f)(a),
$$

whence $\hat{J}(\xi, w) \cdot(\mu, a)=\langle\mu, \xi\rangle+f(a)(w)$, i.e.

$$
J(\mu, a)=(\mu, f(a)) .
$$

Since the action of $6 \times W$ on $(5 \times V)^{*}$ has a momentum mapping, this Lie algebra action is canonical. Hence the action of $G \underset{\Psi}{\times} W$ on $(\mathfrak{G} \underset{\phi}{\times V})^{*}$ is also canonical, thus proving our earlier claim.

Finally, note that $J$ is equivariant. Let us verify this for the group actions, the proof for the Lie algebra actions being similar. We must verify that

$$
\operatorname{Ad}_{(g, w)^{-t}}^{*}(J(\mu, a))=J((g, w) \cdot(\mu, a))
$$

for all $g \in G, w \in W, \mu \in \mathfrak{G}^{*}, a \in V^{*}$. We have by (2.8), (3.1), (3.3), and (3.7)

$$
\begin{aligned}
\operatorname{Ad}_{(g, w)^{-1}}^{*}(J(\mu, a)) & =\operatorname{Ad}_{(g, w)^{-1}}^{*}(\mu, f(a)) \\
& =\left(\operatorname{Ad}_{g-1}^{*} \mu+\psi_{w}^{*} \Psi\left(g^{-1}\right)^{*} f(a), \Psi\left(g^{-1}\right)^{*} f(a)\right) \\
& =\left(\operatorname{Ad}_{g^{-1}}^{*} \mu+\psi_{w}^{*} \Psi\left(g^{-1}\right)^{*} f(a), f\left(\Phi\left(g^{-1}\right)^{*} a\right)\right) \\
& =J\left(\operatorname{Ad}_{g^{-1}}^{*} \mu+\psi_{w}^{*} \Psi\left(g^{-1}\right)^{*} f(a), \Phi\left(g^{-1}\right)^{*} a\right)=J((g, w) \cdot(\mu, a)) .
\end{aligned}
$$

and equivariance of $J$ is proved. By Proposition $1.1 J$ preserves Poisson brackets. We summarize our results in the following.

Theorem 3.1. Let $\Phi: G \rightarrow \operatorname{Aut}(V), \Psi: G \rightarrow \operatorname{Aut}(W)$ be linear representations on the vector spaces $V$ and $W$ and let $f: V^{*} \rightarrow W^{*}$ satisfy $f \circ \Phi(g)^{*}=\Psi(g)^{*} \circ f$ for all $g \in G$. Then the mapping

$$
J:(\mathfrak{6} \underset{\phi}{\times} V)^{*} \rightarrow(6 \mathfrak{6} \underset{\psi}{\times} W)^{*}
$$

given by $J(\mu, a)=(\mu, f(a))$ is canonical.

The Lie algebraic version of this theorem is the following.

Theorem 3.2. Let $\phi:(5) \operatorname{End}(V), \psi:(5) \operatorname{End}(W)$ be linear representations of the Lie algebra $\left(5\right.$ on the vectors spaces $V$ and $W$. Let $f: V^{*} \rightarrow W^{*}$ satisfy $D f(a)(\phi(\xi) * a)$ $=\psi(\xi)^{*}(f(a))$ for all $\xi \in\left(\mathfrak{5}, a \in V^{*}\right.$. Then the mapping

$$
J:(\mathfrak{G} \underset{\phi}{\times} V)^{*} \rightarrow(\mathfrak{5} \underset{\psi}{\times} W)^{*}
$$

given by $J(\mu, a)=(\mu, f(a))$ is canonical

Corollary 3.3. Let $\phi: \mathfrak{G}_{1} \rightarrow$ End $(V), \psi:\left(\mathfrak{G}_{2} \rightarrow\right.$ End $(W)$ be Lie algebra representations and $\alpha: \mathfrak{G}_{2} \rightarrow \mathfrak{G}_{1}$ a Lie algebra homomorphism. Assume that $f: V^{*} \rightarrow W^{*}$ is compatible 
with $\alpha, \phi$, and $\psi$, i.e., it satisfies $D f(a)\left(\phi(\alpha(\eta))^{*} a\right)=\psi(\eta)^{*}(f(a))$ for all $\eta \in \mathfrak{b}_{2}$, $a \in V^{*}$. Then the map

$$
J:\left(\mathfrak{G}_{1} \underset{\phi}{\times} V\right)^{*} \rightarrow\left(\mathfrak{G}_{2} \times W\right)^{*}
$$

given by $J(\mu, a)=\left(\alpha^{*}(\mu), f(a)\right), \mu \in\left(\mathfrak{5}_{1}^{*}, a \in V^{*}\right.$ is canonical, where $\alpha^{*}:\left(\mathfrak{b}_{1}^{*} \rightarrow \mathfrak{G}_{2}^{*}\right.$ is the dual map to $\alpha$.

Proof. Let $\chi=\phi \circ \alpha: \mathfrak{G}_{2} \rightarrow \operatorname{End}(V)$. Clearly $f$ satisfies (3.2) with respect to $\chi$ and $\psi$ so that the map $(v, a) \in\left(\mathfrak{G}_{2} \underset{\chi}{\times} V\right)^{*} \mapsto(v, f(a)) \in\left(\mathfrak{G}_{2} \underset{\psi}{\times} W\right)^{*}$ is canonical. But since the map $(\eta, v) \in \mathfrak{G}_{2} \underset{x}{\times} V \mapsto(\alpha(\eta), v) \in\left(\mathfrak{G}_{1} \underset{\phi}{\times} V\right.$ is a Lie algebra homomorphism, the map $(\mu, a) \in\left(\mathfrak{G}_{1} \underset{\phi}{\times} V\right)^{*} \mapsto\left(\alpha^{*}(\mu), a\right) \in\left(\mathfrak{G}_{2} \times{ }_{x} V\right)^{*}$ is also canonical. The composition of the above mappings equals $J$.

We turn now to the generalization of this result to the case of semidirect products of Lie algebras. If one replaces the vector spaces $V$ and $W$ in Theorem 3.2 by Lie algebras $\mathfrak{S}$ and $\mathfrak{R}$, the mapping $J$ is in general not canonical as the following example shows. If $\mathfrak{\xi}=\{0\}$ condition (3.2) is obviously satisfied and thus the mapping $J$ is canonical if and only if $f: \mathfrak{S}^{*} \rightarrow \mathfrak{\Omega}^{*}$ is canonical. It turns out that this necessary condition is also sufficient. We formulate the next result only in Lie algebraic terms having the applications in mind.

Theorem 3.3. Let $\mathfrak{5}, \mathfrak{H}, \mathfrak{\Omega}$ be Lie algebras and $\phi:(\mathfrak{G} \rightarrow \operatorname{Der}(\mathfrak{H}), \psi:(\mathfrak{H} \rightarrow \operatorname{Der}(\mathfrak{\Omega})$ be Lie algebra homomorphisms. Let $f: \mathfrak{H}^{*} \rightarrow \mathfrak{R}^{*}$ be a canonical map compatible with $\phi$ and $\psi$, i.e.

$$
D f(v)(\phi(\xi) * v)=\psi(\xi)^{*}(f(v))
$$

for all $\xi \in \mathfrak{6}, v \in \mathfrak{S}^{*}$. Then the map

$$
J:(\mathfrak{G} \underset{\phi}{\times} \mathfrak{S})^{*} \rightarrow\left((\mathfrak{G} \underset{\psi}{\times} \mathfrak{\Re})^{*}\right.
$$

given by

$$
J(\mu, v)=(\mu, f(v)), \mu \in \mathfrak{G}^{*}, v \in \mathfrak{S}^{*}
$$

is also canonical.

Proof. A comparison between (2.5) and (2.9) shows that the first three terms in the Lie-Poisson bracket of $(\mathfrak{G} \times \underset{\phi}{H})^{*}$ coincide with the Lie-Poisson bracket of the dual of the semidirect product of $\mathfrak{5}$ with the vector space $\mathfrak{5}$. Hence by Theorem $3.2, J$ preserves these three terms. The fourth term is the Lie-Poisson bracket on $\mathfrak{H}^{*}$. Since $f: \mathfrak{S}^{*} \rightarrow \mathfrak{A}^{*}$ is by hypothesis canonical, $J$ also preserves this term.

Remark. One can further notice that in the above case $J$ is also a momentum map. The action of $\left(\mathfrak{5} \underset{\psi}{\times} \mathfrak{A}\right.$ on $(\mathfrak{G} \underset{\phi}{\times} \mathfrak{G})^{*}$, which has $J$ as an equivariant momentum map is: 


$$
\begin{aligned}
(\xi, \chi) \cdot(\mu, v) & =\left(-(\operatorname{ad} \xi)^{*} \mu+\phi_{D f(v)^{*} \chi}^{*} v,-\phi(\xi)^{*} v-\operatorname{ad}\left(D f(v)^{*} \chi\right)^{*} v\right) \\
& =\left(-(\operatorname{ad} \xi)^{*} \mu+\psi_{\chi}^{*} f(v),-\phi(\xi)^{*} v-\operatorname{ad}\left(D f(v)^{*} \chi\right)^{*} v\right),
\end{aligned}
$$

where $\xi \in \mathfrak{G}, \chi \in \mathfrak{R}, \mu \in \mathfrak{G}^{*}, v \in \mathfrak{H}^{*}$.

The following is proved in a similar way as Corollary 3.3 .

Corollary 3.4. Let $\mathfrak{G}_{1}, \mathfrak{G}_{2}, \mathfrak{H}, \mathfrak{R}$ be Lie algebras and $\phi: \mathfrak{G}_{1} \rightarrow \operatorname{Der}(\mathfrak{S}), \psi:\left(\mathfrak{G}_{2} \rightarrow \operatorname{Der}(\mathfrak{S})\right.$, $\alpha: \mathfrak{G}_{2} \rightarrow \mathfrak{G}_{1}$ be Lie algebra homomorphisms. Let $f: \mathfrak{H}^{*} \rightarrow \mathfrak{R}^{*}$ be a canonical map compatible with $\phi, \psi$, and $\alpha$, that is, satisfying $D f(v)\left(\phi(\alpha(\eta))^{*} v\right)=\psi(\eta)^{*}(f(v))$ for all $\eta \in \mathbb{6}_{2}, v \in \mathfrak{H}^{*}$. Then the map

$$
J:\left(\mathfrak{G}_{1} \underset{\phi}{\times} \mathfrak{S}\right)^{*} \rightarrow\left(\mathfrak{G}_{2} \underset{\psi}{\times} \mathfrak{\Re}\right)^{*}
$$

given by $J(\mu, v)=\left(\alpha^{*}(\mu), f(v)\right), \mu \in \mathfrak{G}_{1}^{*}, v \in \mathfrak{S}^{*}$ is canonical, where $\alpha^{*}:\left(\mathfrak{G}_{1}^{*} \rightarrow\left(\mathfrak{G}_{2}^{*}\right.\right.$ denotes the dual map to $\alpha$.

So far all Lie algebras under consideration could have been also over rings, if we replace everywhere the functions on the dual by polynomials on the dual. The next corollary however deals explicitly with Lie algebras over rings. It is needed in example 6 of the next section.

Let $\mathfrak{R}$ be a Lie algebra and $\phi: \Re \rightarrow \operatorname{End}(V), \psi: \Re \rightarrow \operatorname{End}(W)$ be linear representations. Let $f: V^{*} \rightarrow W^{*}$ be compatible with $\phi$ and $\psi$, i.e. satisfying (3.2). Then $(\mu, a) \mapsto(\mu, f(a))$ is a canonical map of $(\mathfrak{A} \underset{\phi}{\times} V)^{*} \rightarrow(\mathfrak{R} \times \underset{\psi}{\times} W)^{*}$ by Theorem 3.2. Let $R$ be a ring containing the field of scalars of $\mathcal{R}$. Then $R \otimes \Omega$ is a Lie algebra in the obvious way $[r \otimes \xi, s \otimes \eta]=r s \otimes[\xi, \eta]$ for $r, s \in R, \xi, \eta \in \Re$. Let $\Lambda$ be an $R$-module and form $A \otimes V, A \otimes W$, the tensor product being over the field of scalars of $\Omega$. Then there are linear representations $\phi_{R, A}: R \otimes \mathfrak{H} \rightarrow \operatorname{End}(\Lambda \otimes V), \quad \psi_{R, A}: R \otimes \mathfrak{A} \rightarrow$ End $(\Lambda \otimes W)$ given by

$$
\begin{aligned}
\phi_{R, A}(r, \xi) \cdot(\omega, v) & =r \omega \otimes \phi(\xi)(v), \\
\psi_{R, A}(r, \xi) \cdot(\omega, w) & =r \omega \otimes \psi(\xi) w
\end{aligned}
$$

for $r \in R, \xi \in \mathcal{R}, \omega \in \Lambda, v \in V, w \in W$. The map $(\Omega, a) \in(\Lambda \otimes V)^{*} \mapsto(\Omega, f(a)) \in(\Lambda \otimes W)^{*}$, $\Omega \in \Lambda^{*}, a \in V^{*}$ is easily seen to satisfy (3.2) with respect to $\phi_{R, A}$ and $\psi_{R, A}$. Thus the map

$$
\begin{gathered}
(A \otimes v, \Omega \otimes a) \in\left[(R \otimes \mathfrak{R})_{\left.\phi_{R, A} \times(\Lambda \otimes V)\right]^{*} \mapsto}\right. \\
(A \otimes v, \Omega \otimes f(a)) \in\left[(R \otimes \mathfrak{R})_{\left.\psi_{R, A} \times(\Lambda \otimes W)\right]^{*}}\right.
\end{gathered}
$$

is canonical by Thoerem 3.2 .

Now let $\mathfrak{H}$ be another Lie algebra and $\rho: \mathfrak{H} \rightarrow \operatorname{End}(R), \sigma: \mathfrak{H} \rightarrow$ End $(\Lambda)$ be linear representations, where End $(R)$, End $(\Lambda)$ denote the algebras of endomorphisms of $R$ and $\Lambda$ as vector spaces over the field of scalars of $\mathfrak{H}$. In addition assume that $\rho, \sigma$ are compatible with the $R$-module structure of $A$, i.e.

$$
\rho(\xi)(r \omega)=\rho(\xi)(r) \omega+r \sigma(\xi)(\omega)
$$


for $\xi \in \mathfrak{H}, \quad r \in R, \omega \in \mathcal{A}$. Then there are Lie algebra homomorphisms $\chi_{V}: \mathfrak{H} \rightarrow$ $\operatorname{Der}\left(\left(R \otimes \mathcal{R}_{\phi_{R, A}} \times(\Lambda \otimes V)\right), \chi_{W}: \mathfrak{S} \rightarrow \operatorname{Der}\left((R \otimes \mathfrak{R}) \underset{\psi_{R, A}}{\times}(\Lambda \otimes W)\right)\right.$ given by

$$
\begin{aligned}
\chi_{V}(\eta)(r \otimes \xi, \omega \otimes v) & =(\rho(\eta)(r) \otimes \xi, \sigma(\eta)(\omega) \otimes v), \\
\chi_{W}(\eta)(r \otimes \xi, \omega \otimes w) & =(\rho(\eta)(r) \otimes \xi, \sigma(\eta)(\omega) \otimes w),
\end{aligned}
$$

for $\eta \in \mathfrak{H}, r \in R, \xi \in \mathbb{R}, \omega \in \Lambda, v \in V, w \in W$. It is easily seen that the map (3.10) is compatible with the duals of $\chi_{V}$ and $\chi_{W}$ in the sense of (3.8). Thus by Theorem 3.3, the map

$$
\begin{gathered}
(\mu, A \otimes v, \Omega \otimes a) \in\left[\mathfrak{S} \underset{\chi_{V}}{\times}\left((R \otimes \Re) \underset{\phi_{R, A}}{\times}(A \otimes V)\right)\right]^{*} \mapsto \\
(\mu, A \otimes v, \Omega \otimes f(a)) \in\left[\mathfrak{G} \times_{\chi_{W}}\left((R \otimes \mathfrak{S}) \underset{\left.\left.\psi_{R, A} \times(A \otimes W)\right)\right]^{*}}{\times}\right.\right.
\end{gathered}
$$

is canonical. We summarize this result in the following.

Theorem 3.5. Let $\mathfrak{5}, \Omega$ be Lie algebras, $V, W$ vector spaces, $R$ a ring containing the field of scalars of $\mathfrak{S}$ and $\mathfrak{\Omega}$, and $\Lambda$ an $R$-module. Let $\phi: \mathfrak{R} \rightarrow$ End $(V), \psi: \Re \rightarrow$ End $(W)$ be linear representations which naturally induce the linear representations

$$
\phi_{R, \Lambda}: R \otimes \mathfrak{R} \rightarrow \text { End }(\Lambda \otimes V), \psi_{R, \Lambda}: R \otimes R \rightarrow \text { End }(\Lambda \otimes W) .
$$

Let $\rho: \mathfrak{H} \rightarrow$ End $(R), \sigma: \mathfrak{G} \rightarrow \operatorname{End}(\Lambda)$ be linear representations compatible with the $R$ module structure of $\Lambda$. Finally, let $f: V^{*} \rightarrow W^{*}$ be a map satisfying (3.2). Then the map

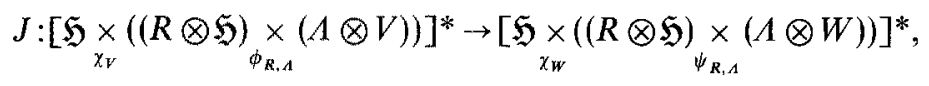

given by

$$
J(\mu, A \otimes v, \Omega \otimes a)=(\mu, A \otimes v, \Omega \otimes f(a))
$$

is a canonical mapping; here $\mu \in \mathfrak{S}^{*}, A \in R^{*}, v \in \mathfrak{R}^{*}, \Omega \in \Lambda^{*}, a \in V^{*}$, and $\chi_{V}, \chi_{W}$ are the Lie algebra homomorphisms of $\mathfrak{H}$ in $\operatorname{Der}((R \otimes \mathfrak{R}) \times(\Lambda \otimes V))$ and $\operatorname{Der}((R \otimes \mathfrak{A}) \times(\Lambda \otimes W))$ cononically induced on the first factors of the tensor products by $\rho$ and $\sigma$ respectively.

\section{Applications}

In this section we consider two physical models: elastodynamics and anisotropic superfluids in the presence of spin. We break up the presentation by treating four mathematical examples first, and then using them in the analysis of the physical systems.

1) Let $V_{1}, V_{2}$ be vector spaces and $\phi_{1}: \mathfrak{5}_{1} \rightarrow \operatorname{End}\left(V_{1}\right) \phi_{2}:\left(\mathfrak{G}_{2} \rightarrow\right.$ End $\left(V_{2}\right)$ be linear representations of two Lie algebras $\mathfrak{G}_{1}$ and $\mathfrak{b}_{2}$. Then there are induced representations

$$
\begin{aligned}
& \phi:\left(\mathfrak{5}_{1} \oplus \mathfrak{G}_{2} \rightarrow \text { End }\left(V_{1} \oplus V_{2}\right)\right. \text { given by } \\
& \phi\left(\xi_{1}, \xi_{2}\right)\left(v_{1}, v_{2}\right)=\left(\phi_{1}\left(\xi_{1}\right) v_{1}, \phi_{2}\left(\xi_{2}\right) v_{2}\right),
\end{aligned}
$$


and

$$
\begin{gathered}
\psi:\left(\boldsymbol{G}_{1} \oplus \boldsymbol{G}_{2} \rightarrow \operatorname{End}\left(V_{1} \otimes V_{2}\right) \quad\right. \text { given by } \\
\psi\left(\xi_{1}, \xi_{2}\right)\left(v_{1} \otimes v_{2}\right)=\phi_{1}\left(\xi_{1}\right) v_{1} \otimes v_{2}+v_{1} \otimes \phi_{2}\left(\xi_{2}\right) v_{2}
\end{gathered}
$$

for $\xi_{i} \in \mathfrak{G}_{i}, \quad v_{i} \in V_{i}, \quad i=1,2$. The map $f:\left(V_{1} \oplus V_{2}\right)^{*} \rightarrow\left(V_{1} \otimes V_{2}\right)^{*}, \quad f\left(a_{1}, a_{2}\right)=$ $a_{1} \otimes a_{2}, a_{i} \in V_{i}, i=1,2$, is easily seen to be compatible with the duals of the foregoing actions. Consequently, by Theorem 3.2 the map

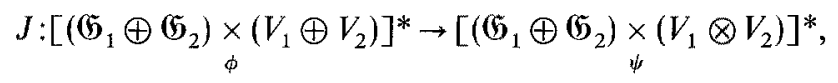

given by

$$
J\left(\mu_{1}, \mu_{2}, a_{1}, a_{2}\right)=\left(\mu_{1}, \mu_{2}, a_{1} \otimes a_{2}\right)
$$

for $\mu_{i} \in \mathfrak{G}_{i}^{*} ; a_{i} \in V_{i}^{*}, i=1,2$, is a canonical mapping. This example can be generalized to any number of factors.

2) A related example occurs when $\boldsymbol{6}_{1}=\mathfrak{6}_{2}=\mathfrak{6}$. Composing the actions $\phi$ and $\psi$ with the diagonal homomorphism $\mathfrak{b} \rightarrow \mathfrak{G} \oplus \mathfrak{b}$, we get the actions $\phi_{1} \oplus \phi_{2}:(\mathfrak{b}$ $\rightarrow$ End $\left(V_{1} \oplus V_{2}\right)$ and $\phi_{1} \otimes \phi_{2}:(5) \operatorname{End}\left(V_{1} \otimes V_{2}\right)$. As before, the map $f:\left(V_{1} \oplus V_{2}\right)^{*}$ $\rightarrow\left(V_{1} \otimes V_{2}\right)^{*}$ given by $f\left(a_{1}, a_{2}\right)=a_{1} \otimes a_{2}$ is compatible with the duals of the above actions. Consequently, by Theorem 3.2, the map

given by

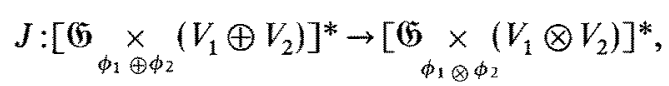

$$
J\left(\mu, a_{1}, a_{2}\right)=\left(\mu, a_{1} \otimes a_{2}\right)
$$

for $\mu \in \mathfrak{G}^{*}, a_{i} \in V_{i}^{*}, i=1,2$, is a canonical mapping. As before, this example has a straightforward generalization to any number of factors.

3) Let $S^{2} V \subset V \otimes V$ denote the symmetric homogeneous polynomials of degree two on $V$. The canonical map (4.2) for $V=V_{1}=V_{2}$ induces another canonical map

given by

$$
\tilde{J}:\left((\mathfrak{G} \underset{\phi}{\times} V)^{*} \rightarrow\left(\mathfrak{G} \underset{\phi \otimes \phi}{\times} S^{2} V\right)^{*},\right.
$$

$$
\widetilde{J}(\mu, a)=(\mu, a \otimes a)
$$

for $\mu \in \mathfrak{G}^{*}, a \in V^{*}$. In fact, the following diagram of canonical maps commutes

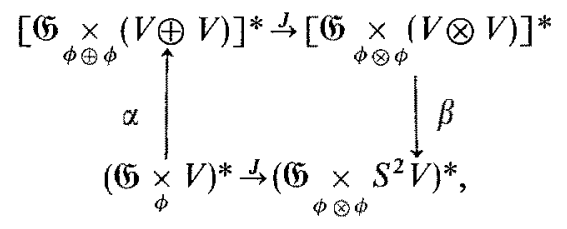

where $\alpha$ is the dual map to the Lie algebra homomorphism $(\xi, v, w) \in(\mathfrak{b} \times(V \otimes V) \mapsto$ $(\xi, v+w) \in \mathfrak{G} \times V$ and $\beta$ is the dual map to the canonical inclusion $6 \times S^{2} V \rightarrow \mathfrak{6}$ $\times(V \otimes V)$. Similarly, the map $J:(6 \underset{\phi}{\times} V)^{*} \rightarrow\left(\mathfrak{6} \underset{\psi}{\times} S^{k} V\right)^{*}$, where $\psi=\phi \otimes \cdots \otimes \phi$, given by $J(\mu, a)=(\mu, a \otimes \cdots \otimes a)$ is canonical; $S^{k} V$ denotes the space of homogeneous 
polynomials of degree $k$ on $V$ and is regarded as a subspace of $V \otimes \cdots \otimes V(k$ factors).

4) Let $f_{i}: V_{i}^{*} \rightarrow W_{i}^{*}$ be compatible with the duals of the representations $\phi_{i}: \mathfrak{G} \rightarrow \operatorname{End}\left(V_{i}\right), \psi_{i}:\left(\mathfrak{5} \rightarrow \operatorname{End}\left(W_{i}\right)\right.$ for each $i=1, \ldots, n$. Since the map $\left(a_{1}, \ldots, a_{n}\right) \mapsto$ $\left(f_{1}\left(a_{1}\right), \ldots, f_{n}\left(a_{n}\right)\right)$ of $\bigoplus_{i=1}^{n} V_{i}^{*}$ to $\bigoplus_{i=1}^{n} W_{i}^{*}$ is compatible with the duals of $\bar{\phi}=$ $\phi_{1} \oplus \ldots \oplus \phi_{n}$ and $\bar{\psi}=\psi \oplus \ldots \oplus \psi_{n}$, the mapping of $\left(\mathfrak{6} \times \underset{\bar{\phi}}{\oplus} \underset{i=1}{n} V_{i}\right)^{*}$ to $\left(\mathfrak{b} \underset{\bar{\phi}}{\times} \bigoplus_{i=1}^{n} W_{i}\right)^{*}$ given by

$$
\left(\mu, a_{1}, \ldots, a_{n}\right) \mapsto\left(\mu, f_{1}\left(a_{1}\right), \ldots, f_{n}\left(a_{n}\right)\right)
$$

is canonical.

Assume now that all $W_{i}=W, \psi_{i}=\psi, i=1, \ldots, n$ and compose the mapping (4.4) with the dual of the Lie algebra homomorphism $\underset{\psi}{\mathfrak{G}} \times \underset{\psi}{\times} \rightarrow \mathfrak{G} \underset{\bar{\psi}}{\times} \oplus W_{i}$, $(\xi, w) \mapsto(\xi, w, \ldots, w)$. In this way we get a canonical map

$$
J:\left(\mathfrak{G} \underset{\bar{\phi}}{\times} \underset{i=1}{\oplus} V_{i}\right)^{*} \rightarrow(\mathfrak{G} \underset{\psi}{\times} W)^{*},
$$

given by

$$
J\left(\mu, a_{1}, \ldots, a_{n}\right)=\left(\mu, f_{1}\left(a_{1}\right)+\cdots+f_{n}\left(a_{n}\right)\right),
$$

$\mu \in \mathfrak{G}^{*}, a_{i} \in V_{i}^{*}, i=1, \ldots, n$.

5) Elasticity. The map (4.5) arises in the Poisson bracket formulation of elasticity. The following is the relevant material from [5]. The equations of elastodynamics in $\mathbb{B}^{n}$ are Hamiltonian on the dual of the semidirect product Lie algebra

$$
\mathscr{X}\left(\mathbb{R}^{n}\right) \times\left[\mathscr{F}\left(\mathbb{R}^{n}\right) \oplus \mathscr{F}\left(\mathbb{R}^{n}\right) \oplus_{i=1}^{n} A^{n-1}\left(\mathbb{R}^{n}\right)(i)\right]
$$

where a vector field in $\mathscr{X}\left(\mathbb{R}^{n}\right)$ acts by Lie derivative on every factor. The corresponding duals are:

- $\mathscr{X}\left(\mathbb{R}^{\prime \prime}\right)$ has as dual the one-form densities; the pairing is the action of one-form densities on vector fields followed by integration; the physical variables are $M_{i}, i$ $=1, \ldots, n$, the components of the momentum density, which as vector field equals $\rho v$, $\rho$ being the density of the material and $v$ the velocity field of the displacement; - $\mathscr{F}\left(\mathbb{R}^{n}\right)$ has as dual the densities on $\mathbb{R}^{n}$; the pairing is multiplication of a function by a density followed by integration; the physical variables are the density of the material $\rho$ for the first factor and the entropy density $\sigma$ for the second factor;

- $\underset{i=1}{\oplus} A^{n-1}\left(\mathbb{R}^{n}\right)(i)$ has as dual $\underset{i=1}{\oplus} A^{1}\left(\mathbb{R}^{n}\right)(i)$; the pairing on each summand is the wedge product of an $(n-1)$-form with a one-form followed by integration; the physical variables on the $i^{\text {th }}$ summand are the $n$ components $F_{i j}$ of the deformation gradient $F, j=1, \ldots, n$.

The bracket of two functions $f, g$ depending on the variables $M_{i}, \rho, \sigma, F_{i j}$ is given 
by (see (80)-(86) in [5])

$$
\begin{gathered}
\{f, g\}\left(M_{i}, \rho, \sigma, F_{i j}\right)=\int d^{n} x\left\{\frac{\delta g}{\delta M_{i}}\left(M_{j} \partial_{i}+\partial_{j} M_{i}\right) \frac{\delta f}{\partial M_{j}}\right. \\
+\frac{\partial g}{\partial M_{i}}\left(\rho \partial_{i} \frac{\delta f}{\delta \rho}+\sigma \partial_{i} \frac{\delta f}{\delta \sigma}\right)+\left(\frac{\delta g}{\delta \rho} \partial_{i} \rho+\frac{\delta g}{\delta \sigma} \partial_{i} \sigma\right) \frac{\delta f}{\delta M_{i}} \\
\left.+\frac{\delta g}{\delta M_{i}}\left(\partial_{k} F_{s i}-F_{s i, k}\right) \frac{\delta f}{\delta F_{s k}}+\frac{\delta g}{\delta F_{s i}}\left(F_{s k} \partial_{i}+F_{s i, k}\right) \frac{\delta f}{\delta M_{k}}\right\},
\end{gathered}
$$

where $\partial_{k}=\partial / \partial x_{k}$ and $(\cdot)_{, k}=\partial(\cdot) / \partial x_{k}$.

If one uses the standard hypothesis of material frame indifference, then the stored energy function, and hence the Hamiltonian, depends only on the Cauchy stress tensor $e=F^{t} F$ (see e.g. [2]). In this case the equations of elastodynamics become Hamiltonian on the dual of the Lie algebra $\mathscr{X}\left(\mathbb{R}^{n}\right) \times\left[\mathscr{F}\left(\mathbb{R}^{n}\right) \oplus\right.$ $\left.\mathscr{F}\left(\mathbb{R}^{n}\right) \oplus\left(S^{2}\left(\mathscr{X}\left(\mathbb{R}^{n}\right)\right) \otimes \Lambda^{n}\left(\mathbb{R}^{n}\right)\right)\right]$, where $\mathscr{X}\left(\mathbb{R}^{n}\right)$ acts by the Lie derivative on every factor and $S^{2}\left(\mathscr{X}\left(\mathbb{R}^{n}\right)\right) \otimes A^{n}\left(\mathbb{R}^{n}\right)$ are the contravariant symmetric two-tensor densities on $\mathbb{R}^{n}$. The dual of this space is the vector space of covariant symmetric two-tensors $S^{2}\left(\Lambda^{1}\left(\mathbb{R}^{n}\right)\right)$ and the corresponding physical variable is the Cauchy stress tensor $e$. The bracket of two functions $f, g$ depending on the variables $\left(M_{i}, \rho, \sigma, e_{i j}\right)$ is given by (see (88) in [5])

$$
\begin{aligned}
\{f, g\}\left(M_{i}, \rho, \sigma, e_{i j}\right)= & \{f, g\}_{M_{i}, \rho, \sigma}+\int d^{n} x\left\{\frac{\delta g}{\delta M_{i}}\left(2 \partial_{k} e_{i \beta}-e_{k \beta, i}\right) \frac{\delta f}{\delta e_{k \beta}}\right. \\
& \left.+\frac{\delta g}{\delta e_{i \beta}}\left(2 e_{\beta k} \partial_{i}+e_{\beta i, k}\right) \frac{\partial f}{\partial M_{k}}\right\},
\end{aligned}
$$

where $\{f, g\}_{M_{i, \rho}, \sigma}$ denotes the part of the bracket (4.6) independent of $F_{i j}$ given by its first two rows; the corresponding bracket in terms of the finger deformation tensor is given in [8].

The passage from (4.6) to (4.7) is achieved by the map

$$
e_{i j}=\sum_{k=1}^{n} F_{k i} F_{k j}, \text { i.e., } e=F^{t} F,
$$

defining the Cauchy stress tensor in terms of the deformation gradient. Denoting by $A_{k}$ the one-form on $\mathbb{R}^{n}$ having components $F_{k i}, i=1, \ldots, n,(4.8)$ is a map of $\left[\oplus_{i=1}^{n} A^{n-1}\left(\mathbb{R}^{n}\right)(\mathrm{i})\right]^{*} \rightarrow S^{2}\left(\Lambda^{1}\left(\mathbb{R}^{n}\right)\right)$ given by $\left(A_{1}, \ldots, A_{n}\right) \mapsto A_{1} \otimes A_{1}+\ldots+A_{n} \otimes A_{n}$. Take now in $(4.5) \quad\left(5=\mathscr{X}\left(\mathbb{R}^{n}\right), V_{1}=\ldots=V_{n}=\Lambda^{n-1}\left(\mathbb{R}^{n}\right), W=S^{2}\left(\mathscr{X}\left(\mathbb{R}^{n}\right)\right) \otimes \Lambda^{n}\left(\mathbb{R}^{n}\right)\right.$, $f_{1}=\ldots=f_{n}=f, f(A)=A \otimes A, A \in \Lambda^{1}\left(\mathbb{R}^{n}\right)$. By $(4.5)$ the mapping $\left(M_{i}, F_{i j}\right) \mapsto\left(M_{i}, e_{i j}\right)$ is canonical. Hence by (4.4) the map $\left(M_{i}, \rho, \sigma, F_{i j}\right) \mapsto\left(M_{i}, \rho, \sigma, e_{i j}\right)$ is also canonical. We summarize this result in the following.

Theorem 4.1. The mapping $\left(M_{i}, \rho, \sigma, F_{i j}\right) \mapsto\left(M_{i}, \rho, \sigma, e_{i j}=\left(F^{t} F\right)_{i j}\right)$, which on the last component represents the definition of the Cauchy stress tensor, is a canonical mapping between the Poisson structures (4.6) and (4.7) associated to the equations of elastodynamics. 
6) Anisotropic superfluid with spin. In [6] it has been shown that the equations of motion for an anisotropic superfluid with spin are Hamiltonian on the direct sum of two Lie algebras whose description follows. We shall use the notations of Theorem 3.5 .

The first summand is a semidirect product $\mathfrak{H} \times[(R \otimes \mathfrak{R}) \times(A \otimes V)]$, where $\mathfrak{S}=\mathscr{X}\left(\mathbb{R}^{n}\right), R=\mathscr{F}\left(\mathbb{R}^{n}\right), \Lambda=\Lambda^{n}\left(\mathbb{R}^{n}\right), \mathfrak{R}=\boldsymbol{6} \oplus \boldsymbol{G} \oplus \mathbb{R}$, where 6 is a finite dimensional Lie algebra representing the internal symmetry algebra of the superfluid (for ${ }^{3} \mathrm{He}-A, \quad \mathfrak{G}=\mathrm{so}(3)$ ), and $V=\mathfrak{6} \oplus \mathfrak{G} \oplus \mathfrak{6}$. The relevant actions are the following: $\mathfrak{R}$ acts on $V$ by

$$
\left(\xi_{1}, \xi_{2}, \alpha\right) \cdot\left(\eta_{1}, \eta_{2}, \eta_{3}\right)=\left(\left[\xi_{1}, \eta_{1}\right],\left[\xi_{2}, \eta_{2}\right]+2 m \alpha \eta_{3},\left[\xi_{2}, \eta_{3}\right]-2 m \alpha \eta_{2}\right)
$$

for $\xi_{1}, \xi_{2}, \eta_{1}, \eta_{2}, \eta_{3} \in \mathfrak{6}, \alpha \in \mathbb{R}$, where $m$ is an arbitrary constant (the mass of the ${ }^{3} \mathrm{He}$ atom in physics). Finally, $\mathfrak{H}=\mathscr{X}\left(\mathbb{R}^{n}\right)$ acts on $R$ and $\Lambda$ by Lie derivative.

The second summand is the semidirect product $\mathfrak{S} \times \underset{\rho}{R}=\mathscr{X}\left(\mathbb{R}^{n}\right) \times \mathscr{F}\left(\mathbb{R}^{n}\right)$, where $\mathfrak{S}$ acts on $R$ by Lie derivative.

The description of the dual of this Lie algebra is the following. The first summand:

- $\mathscr{X}\left(\mathbb{R}^{n}\right)$ has as dual the one form densities; the pairing is the action of one-form densities on vector fields followed by integration; the physical variable is the momentum density $M$ of the superfluid;

- in $(R \otimes \Re)^{*}$ the three summands have the following interpretation:

a) $R \otimes \mathbb{6}$ has dual $A^{n}\left(\mathbb{R}^{n}\right) \otimes\left(\mathfrak{G}^{*}\right.$; the physical variable $S$ is called the or bital spin density;

b) $R \otimes\left(5\right.$ has dual $A^{n}\left(\mathbb{R}^{n}\right) \otimes\left(5^{*}\right.$; the physical variable $L$ is called the orbital angular momentum density;

c) $(R \otimes \mathbb{R})^{*}$ has dual $A^{n}\left(\mathbb{R}^{n}\right)$; the physical variable is the density $\rho$ of the superfluid.

- in $(A \otimes V)^{*}$ the three summands have the following interpretation:

a) $\Lambda \otimes\left(5\right.$ has dual $\bar{F}\left(\mathbb{R}^{n}\right) \otimes \mathfrak{G}^{*}$; the physical variable $n$ is called the spin vector;

b) The following two summands in the dual are again $\mathscr{F}\left(\mathbb{P}^{n}\right) \otimes \mathfrak{G}^{*}$ and the two physical variables are the first and second order parameter $\psi^{1}$ and $\psi^{2}$.

The second summand:

- $\mathscr{X}\left(\mathbb{R}^{n}\right)$ has as dual the one-form densities, and again the physical variable is the momentum density;

- $\mathscr{F}\left(\mathbb{R}^{n}\right)$ has as dual $A^{n}\left(\mathbb{R}^{n}\right)$; the physical variable is the entropy density $s$.

The Poisson bracket of two functions $F, G$ depending on $\left(M, S, L, \rho, n, \psi^{1}, \psi^{2}, s\right)$ equals (see (23) in [6])

$$
\begin{aligned}
\{F, G\}= & \int d^{n} x\left\{\left[\frac{\delta G}{\delta s} \partial_{l} s+\frac{\delta G}{\delta n_{\mu}} n_{\mu, l}+\frac{\delta G}{\delta \psi_{\mu}^{1}} \psi_{\mu, l}^{1}+\frac{\delta G}{\delta \psi_{\mu}^{2}} \psi_{\mu, l}^{2}\right.\right. \\
& \left.+\frac{\delta G}{\delta L_{\mu}} \partial_{l} L_{\mu}+\frac{\delta G}{\delta S_{\mu}} \partial_{l} S_{\mu}+\frac{\delta G}{\delta \rho} \partial_{l} \rho+\frac{\delta G}{\delta M_{k}}\left(M_{l} \partial_{k}+\partial_{l} M_{k}\right)\right] \frac{\delta F}{\delta M_{l}}
\end{aligned}
$$




$$
\begin{aligned}
& +\frac{\delta G}{\delta M_{k}}\left[s \partial_{k} \frac{\partial F}{\partial s}-n_{\mu, k} \frac{\delta F}{\delta n_{\mu}}-\psi_{\mu, k}^{1} \frac{\delta F}{\delta \psi_{\mu}^{1}}-\psi_{\mu, k}^{2} \frac{\delta F}{\delta \psi_{\mu}^{2}}+L_{\mu} \partial_{k} \frac{\delta F}{\delta L_{\mu}}\right. \\
& \left.+S_{\mu} \partial_{k} \frac{\delta F}{\delta S_{\mu}}+\rho \partial_{k} \frac{\delta F}{\delta \rho}\right]+2 m\left(\frac{\delta G}{\delta \psi_{\mu}^{2}} \psi_{\mu}^{1}-\frac{\delta G}{\delta \psi_{\mu}^{2}} \psi_{\mu}^{2}\right) \frac{\delta F}{\delta \rho} \\
& +2 m \frac{\delta G}{\delta \rho}\left(\psi_{\mu}^{2} \frac{\delta F}{\delta \psi_{\mu}^{1}}-\psi_{\mu}^{1} \frac{\delta F}{\delta \psi_{\mu}^{2}}\right)+t_{v \mu}^{\sigma}\left[\left(\frac{\delta G}{\delta n_{\mu}} \frac{\delta F}{\delta S_{v}}+\frac{\delta G}{\delta S_{\mu}} \frac{\delta F}{\delta n_{v}}\right) n_{\sigma}\right. \\
& +\left(\frac{\delta G}{\delta \psi_{\mu}^{1}} \psi_{\sigma}^{1}+\frac{\delta G}{\delta \psi_{\mu}^{2}} \psi_{\sigma}^{2}\right) \frac{\delta F}{\delta L_{v}}+\frac{\delta G}{\delta L_{\mu}}\left(\psi_{\sigma}^{1} \frac{\delta F}{\delta \psi_{v}^{1}}+\psi_{\sigma}^{2} \frac{\delta F}{\delta \psi_{v}^{2}}\right. \\
& \left.\left.\left.+L_{\sigma} \frac{\delta F}{\delta L_{v}}\right)+\frac{\delta G}{\delta S_{\mu}} \frac{\delta F}{\delta S_{v}} S_{\sigma}\right]\right\}
\end{aligned}
$$

where $t_{v \mu}^{\sigma}$ are minus the structure constants of $\mathbf{6}$.

The order parameters $\psi^{1}$ and $\psi^{2}$ are the real and imaginary parts of the complex order parameter $\psi=\psi^{1}+i \psi^{2}$ used in physics. However, other order parameters are sometimes in use in the description of superfluids (see e.g. [7]): $A_{\mu \nu}^{1}=n_{\mu} \psi_{v}^{1}, A_{\mu \nu}^{2}=n_{\mu} \psi_{v}^{2}$. As was observed in [6] there exists a counterpart of the bracket (4.9) in terms of the variables $A^{1}, A^{2}$ instead of $n, \psi^{1}, \psi^{2}$ (with all other variables unchanged). Let us show that this fact follows directly from the theorems of Sect. 3. The map $V^{*} \rightarrow W^{*}=\left[\mathfrak{6} \otimes(\mathfrak{6} \otimes(\mathfrak{6})]^{*}=\right.$ $\left(\mathfrak{G}^{*} \otimes \mathfrak{G}^{*}\right) \otimes\left(\mathfrak{G}^{*} \otimes \mathfrak{G}^{*}\right)$, such that the associated map $\mathscr{F}\left(\mathbb{R}^{n}\right) \otimes V^{*} \rightarrow$ $\mathscr{F}\left(\mathbb{R}^{n}\right) \otimes W^{*}$ is given by $\left(n, \psi^{1}, \psi^{2}\right) \mapsto\left(A^{1}, A^{2}\right), A^{1}=n \otimes \psi^{1}, A^{2}=n \otimes \psi^{2}$ is canonical by Theorem 3.2 and formula (4.4). Now apply Theorem 3.5 to get a canonical map

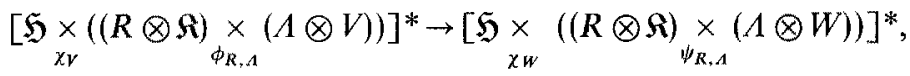

$$
\begin{aligned}
& \left(M, S, L, \rho, n, \psi^{1}, \psi^{2}\right) \mapsto\left(M, S, L, \rho, A^{1}, A^{2}\right) .
\end{aligned}
$$

Finally apply (4.4) to conclude that $\left(M, S, L, \rho, n, \psi^{1}, \psi^{2}, s\right) \mapsto\left(M, S, L, \rho, A^{1}, A^{2}, s\right)$ is canonical. The bracket in these new variables being a Lie-Poisson bracket can be now written directly for any two functions $F, G$ depending on $\left(M, S, L, \rho, n, A^{1}, A^{2}, s\right)$ :

$$
\begin{aligned}
\{F, G\}= & \int d^{n} x\left\{\left[\frac{\delta G}{\delta S} \partial_{l} s+\frac{\delta G}{\delta A_{\mu \nu}^{1}} A_{\mu \nu, l}^{1}+\frac{\delta G}{\delta A_{\mu \nu}^{2}} A_{\mu \nu, l}^{2}\right.\right. \\
& \left.+\frac{\delta G}{\delta L_{\mu}} \partial_{l} L_{\mu}+\frac{\delta G}{\delta S_{\mu}} \partial_{l} S_{\mu}+\frac{\delta G}{\delta \rho} \partial_{l} \rho+\frac{\delta G}{\delta M_{k}}\left(M_{l} \partial_{k}+\partial_{l} M_{k}\right)\right] \frac{\delta F}{\delta M_{l}} \\
& +\frac{\delta G}{\delta M_{k}}\left[s \partial_{k} \frac{\delta F}{\delta S}-A_{\mu \nu, k}^{1} \frac{\delta F}{\delta A_{\mu \nu}^{1}}-A_{\mu \nu, k}^{2} \frac{\delta F}{\delta A_{\mu \nu}^{2}}+L_{\mu} \partial_{k} \frac{\delta F}{\delta L_{\mu}}\right. \\
& \left.+S_{\mu} \partial_{k} \frac{\delta F}{\delta S_{\mu}}+\rho \partial_{k} \frac{\delta F}{\delta \rho}\right]+2 m\left(\frac{\delta G}{\delta A_{\mu \nu}^{2}} A_{\mu v}^{1}-\frac{\delta G}{\delta A_{\mu \nu}^{1}} A_{\mu \nu}^{2}\right) \frac{\delta F}{\delta \rho} \\
& +2 m \frac{\delta G}{\delta \rho}\left(A_{\mu \nu}^{2} \frac{\delta F}{\delta A_{\mu \nu}^{1}}-A_{\mu v}^{1} \frac{\delta F}{\delta A_{\mu \nu}^{2}}\right)
\end{aligned}
$$




$$
\begin{aligned}
& +t_{\varepsilon \alpha}^{\sigma}\left[\frac{\delta G}{\delta S_{\alpha}}\left(S_{\sigma} \frac{\delta F}{\delta S_{\varepsilon}}+A_{\sigma \mu}^{1} \frac{\delta F}{\delta A_{\varepsilon \mu}^{1}}+A_{\sigma \mu}^{2} \frac{\delta F}{\delta A_{\varepsilon \mu}^{2}}\right)\right. \\
& +\frac{\partial G}{\partial L_{\alpha}}\left(L_{\sigma} \frac{\delta F}{\delta L_{\varepsilon}}+A_{\mu \sigma}^{1} \frac{\delta F}{\delta A_{\mu \varepsilon}^{1}}+A_{\mu \sigma}^{2} \frac{\delta F}{\delta A_{\mu \varepsilon}^{2}}\right) \\
& \left.\left.+\frac{\delta F}{\delta L_{\varepsilon}}\left(A_{\mu \sigma}^{1} \frac{\delta G}{\delta A_{\mu \alpha}^{1}}+A_{\mu \sigma}^{2} \frac{\delta G}{\delta A_{\mu \alpha}^{2}}\right)+\frac{\delta F}{\delta S_{\varepsilon}}\left(A_{\sigma \nu}^{1} \frac{\delta G}{\delta A_{\alpha \nu}^{1}}+A_{\sigma \nu}^{2} \frac{\delta G}{\delta A_{\alpha \nu}^{2}}\right)\right]\right\} .
\end{aligned}
$$

Thus we have proved the following.

Theorem 4.2. With the foregoing notation, the mapping $\left(M, S, L, \rho, n, \psi^{1}, \psi^{2}, s\right) \mapsto(M$, $\left.S, L, \rho, A^{1}, A^{2}, s\right), A^{i}=n \otimes \psi^{i}, i=1,2$ is a canonical mapping between the Lie-Poisson structures given by (4.9) and (4.10) describing the Hamiltonian structure of the equations of motion of an anisotropic superfluid with spin.

\section{References}

1. Abraham, R., Marsden, J.: Foundations of mechanics. Reading, MA: Addison-Wesley 1978

2. Eringen, A. C.: Mechanics of continua. Huntington, NY: Robert Krieger Publishing Co. 1980

3. Guillemin, V., Sternberg, S.: The moment map and collective motion. Ann. Phys. 127, 220-253 (1980)

4. Hochschild, G.: The structure of Lie groups. San Francisco: Holden-Day 1965

5. Holm, D., Kupershmidt, B.: Poisson brackets and Clebsch representations for magnetohydrodyamics, multifluid plasmas, and elasticity. Physica D (in press)

6. Holm, D., Kupershmidt, B. : Poisson structures of superfluids. Phys. Lett. 91A, 425-430 (1982)

7. Leggett, A. J.: A theoretical description of the new phases of liquid He. Rev. Mod. Phys. 47, 331 (1975)

8. Marsden, T., Ratiu, T., Weinstein, A.: Semidirect products and reduction in mechanics. Trans. Am. Math. Soc. (in press)

9. Weinstein, A.: Lectures on sympletic manifolds. CBMS Conf. Series No. 27, Providence, RI: Am. Math. Soc. 1977

10. Weinstein, A.: The local structure of Poisson manifolds. Diff. Geom. (in press)

Communicated by A. Jaffe

Received November 30, 1982; in revised form March 10, 1983 